\title{
$\beta$-Adrenergic Receptors Regulate the Acquisition and Consolidation Phases of Aversive Memory Formation Through Distinct, Temporally Regulated Signaling Pathways
}

\author{
Hillary C Schiff', Joshua P Johansen ${ }^{2,3}$, Mian Hou', David EA Bush', Emily K Smith', JoAnna E Klein', \\ Joseph E LeDoux ${ }^{1,4,5}$ and Robert M Sears*,1,5,6 \\ 'Center for Neural Science, New York University, New York, NY, USA; ${ }^{2}$ RIKEN Brain Science Institute, Laboratory for Neural Circuitry of Memory, \\ Wako-shi, Saitama, Japan; ${ }^{3}$ Department of Life Sciences, Graduate School of Arts and Sciences, University of Tokyo, Tokyo, Japan; ${ }^{4}$ Department of \\ Psychology, New York University, New York, NY, USA; ${ }^{5}$ Emotional Brain Institute, Nathan Kline Institute for Psychiatric Research, Orangeburg, NY, \\ USA; ${ }^{6}$ Department of Child and Adolescent Psychiatry, New York University School of Medicine, New York, NY, USA
}

\begin{abstract}
Memory formation requires the temporal coordination of molecular events and cellular processes following a learned event. During Pavlovian threat (fear) conditioning (PTC), sensory and neuromodulatory inputs converge on post-synaptic neurons within the lateral nucleus of the amygdala (LA). By activating an intracellular cascade of signaling molecules, these G-protein-coupled neuromodulatory receptors are capable of recruiting a diverse profile of plasticity-related proteins. Here we report that norepinephrine, through its actions on $\beta$-adrenergic receptors ( $\beta$ ARs), modulates aversive memory formation following PTC through two molecularly and temporally distinct signaling mechanisms. Specifically, using behavioral pharmacology and biochemistry in adult rats, we determined that $\beta$ AR activity during, but not after PTC training initiates the activation of two plasticity-related targets: AMPA receptors (AMPARs) for memory acquisition and short-term memory and extracellular regulated kinase (ERK) for consolidating the learned association into a long-term memory. These findings reveal that $\beta$ AR activity during, but not following PTC sets in motion cascading molecular events for the acquisition (AMPARs) and subsequent consolidation (ERK) of learned associations.

Neuropsychopharmacology (2017) 42, 895-903; doi:I0.1038/npp.2016.238; published online I6 November 2016
\end{abstract}

\section{INTRODUCTION}

Associative learning in response to aversive stimuli allows animals to predict and avoid dangerous situations and is therefore essential to survival. Pavlovian threat conditioning (PTC) is a well-studied paradigm of associative memory formation in which a previously neutral conditioned stimulus (CS), such as an auditory tone, predicts an aversive unconditioned stimulus (US), usually a footshock. Once learned, the CS elicits species-specific defensive responses such as freezing in anticipation of the US (Fanselow and Poulos, 2005; LeDoux, 2014). Although traditionally called Pavlovian fear conditioning, we prefer PTC to avoid the implication that a subjective state of fear is responsible for the responses elicited by the CS (LeDoux, 2014).

Memory formation for the CS-US association in PTC depends on synaptic plasticity within the LA (Rogan et al, 1997; McKernan and Shinnick-Gallagher, 1997), and this plasticity is regulated by neuromodulators including norepinephrine (NE; Bailey et al, 2000; Berridge and

\footnotetext{
*Correspondence: Dr RM Sears, Center for Neural Science, New York University, 4 Washington Place, New York, NY 10003, USA, Tel: 212998 393।, Fax: 212995 4704, E-mail: robert.sears@nyu.edu Received 25 January 2016; revised 5 October 2016; accepted 7 October 20 16; accepted article preview online 20 October 2016
}

Waterhouse, 2003; Sears et al, 2014; Uematsu et al, 2015). $\mathrm{NE}$ is required for PTC memory formation (Fendt et al, 1994; Schulz et al, 2002; but see Murchison et al, 2004), operating at least partially on $\beta$-adrenergic receptors ( $\beta$ ARs), which are required specifically during the acquisition phase, when CS-US pairings occur (Bush et al, 2010; Johansen et al, 2014).

NE modulates synaptic transmission, plasticity, and learning (Hatfield and McGaugh, 1999; Schulz et al, 2002; Tully et al, 2007; Faber et al, 2008) by binding to G-proteincoupled receptors, particularly $\beta$ ARs. Subsequent accumulation of the second messenger cyclic adenosine monophosphate (cAMP) activates myriad downstream effectors including cAMP-dependent protein kinase (PKA) and extracellular regulated kinase (ERK). Despite an extensive knowledge of the diversity of downstream effectors recruited by cAMP signaling in vitro, we do not know which targets are responsible when $\beta \mathrm{AR}$ activity modulates memory formation in vivo. Further, we do not understand how the relative timing of $\beta$ AR's targets contributes to different phases of memory formation. This information is crucial for an understanding of the adrenergic system's function in memory formation, as well as for pharmacological targeting of these effectors in a time-effective manner in the clinic. 
Because footshocks and other acute stressors phasically activate NE-producing neurons in the locus coeruleus (Ennis et al, 1992; Chen and Sara, 2007) and lead to NE release in the amygdala (Quirarte et al, 1998), we hypothesized that $\beta \mathrm{AR}$ activity and subsequent intracellular signaling are required during acquisition when shocks are administered. Classically, effects on learning (acquisition) vs post-learning consolidation processes can be distinguished when a manipulation is effective before $v s$ immediately after training (McGaugh, 1966; Rodrigues et al, 2004; McGaugh and Roozendaal, 2009). We previously showed that $\beta \mathrm{AR}$ antagonism in the LA before PTC training impairs learning, whereas the same manipulation following PTC does not affect memory formation (Bush et al, 2010). Although these findings implicate $\beta \mathrm{AR}$ activity in acquisition, they do not exclude the possibility that $\beta \mathrm{AR}$ activation during training initiates downstream effectors required for later phases of memory formation.

We examined the intracellular targets of $\beta \mathrm{AR}$ activity and the timing of their activation relative to PTC training using the $\beta \mathrm{AR}$ agonist isoproterenol (ISO). First we show that $\beta \mathrm{AR}$ activation in the LA during, but not after, PTC training is sufficient to enhance both short-term memory (STM) and long-term memory (LTM), but that stimulation of $\beta$ ARs promotes acquisition and consolidation via distinct signaling elements. We found that $\beta \mathrm{AR}$ activity during training recruits calcium-permeable AMPARs (CP-AMPARs) in the $\mathrm{LA}$; this process is required for acquisition. $\beta$ ARs, while active only during acquisition, also lead to later activation of ERK which is necessary for consolidation processes, but not for acquisition. Thus, $\beta \mathrm{AR}$ stimulation during acquisition of Pavlovian associations initiates a signaling cascade in LA neurons producing a distinct intracellular molecular context for different phases of memory formation.

\section{MATERIALS AND METHODS}

\section{Animals}

Adult male Sprague-Dawley rats weighing 250-275 g were obtained from Hilltop Laboratory Animals (Scottdale, PA, USA). Rats were individually housed in transparent Nalgene cages and maintained on a 12/12 h light/dark cycle within a temperature- and humidity-controlled environment. Rats had ad libitum access to food and water. All experiments were conducted in accordance with the National Institutes of Health Guide for the Care and Use of Experimental Animals and were approved by the New York University Animal Care and Use Committee.

\section{Stereotaxic Surgery}

Rats were anesthetized with a combination of ketamine (Ketaject; $100 \mathrm{mg} / \mathrm{kg}$, intraperitoneally (i.p.)) and xylazine (Xyla-Ject; $6.0 \mathrm{mg} / \mathrm{kg}$ i.p.), with supplemental boosters as needed. They were placed in a stereotaxic apparatus holding stainless steel guide cannulae (22 gauge; Plastics One, Roanoke, VA, USA), fitted with 28-gauge internal infusion cannula with $1.2 \mathrm{~mm}$ extension. The cannulae were lowered into the LA at the following coordinates from bregma: $-3 \mathrm{~mm}$ anteroposterior; $+5.4 \mathrm{~mm}$ mediolateral; and $-8.0 \mathrm{~mm}$ dorsoventral. The cannulae were secured to the skull with surgical screws and acrylic dental cement. The internal infusion cannulae were removed and replaced with 28-gauge dummy cannulae until drug infusion. Rats recovered over 5-7 days between surgery and behavioral manipulations. Cannulae placements can be seen in Supplementary Figure S1.

\section{Microinfusions and Drug Preparation}

Microinfusions took place as previously described (eg, Bush et al, 2010) and details are described in the Supplementary Methods. All drugs were dissolved in $0.25 \mu \mathrm{l}$ vehicle and all infusions were bilateral. Rats received $1.25 \mu \mathrm{g}$ per side of (-)-isoproterenol hydrochloride (ISO; Sigma-Aldrich, St Louis, MO, USA) dissolved in artificial cerebrospinal fluid (ACSF). ISO was dissolved immediately prior to infusion, which occurred $15 \mathrm{~min}$ before behavioral testing. For ERK inhibition, rats received $1 \mu \mathrm{g}$ per side SL327 (SL327, Z-\& E- $\alpha$-(Amino-((4-aminophenyl)thio)methylene)-2-(trifluoromethyl)benzeneacetonitrile; EMD Millipore, Kankakee, IL) dissolved in ACSF with $10 \%$ or $50 \%$ dimethyl sulfoxide (DMSO) or a cocktail of $1.25 \mu \mathrm{g}$ ISO plus $1 \mu \mathrm{g}$ SL327 dissolved in ACSF with 10\% DMSO 20 min prior to PTC training. For CP-AMPAR inhibition, rats received $1.25 \mu \mathrm{g}$ per side NASPM (1-Naphthylacetyl spermine trihydrochloride; Sigma), an inhibitor of CP-AMPARs, dissolved in ACSF (Ding et al, 2014). For these studies, animals received 2 infusions: NASPM or ACSF $1 \mathrm{~h}$ before training and ISO or ACSF 15 min before training.

\section{PTC Apparatus and Procedures}

PTC occurred as previously described (eg Bush et al, 2010; see Supplementary Methods for details). Briefly, on day 1, rats underwent a 30 -min habituation period to the training context (context A). On day 2, rats underwent PTC training in context A consisting of three conditioning trials. The CS was a $30-\mathrm{s}, 5 \mathrm{kHz}, 80 \mathrm{~dB}$ SPL sine-wave tone, co-terminating with a 1-second, $0.4 \mathrm{~mA}$ footshock. Testing was conducted 3 or $48 \mathrm{~h}$ after training for STM or LTM, respectively, in context B.

\section{Western Blotting}

Details of western blotting procedures can be found in the Supplementary Methods. Briefly, lateral amygdalae were dissected from frozen brains using a freezing microtome. Samples were prepared for western blot by homogenizing in $1 \%$ SDS with protease and phosphatase inhibitor cocktails 1 and 2 (Sigma) in the buffer. $15 \mu \mathrm{g}$ of protein was loaded into Invitrogen (Carlsbad, CA) Tris-Glycine Midi gels (4-12\%). Proteins were transferred from SDS-PAGE gels to a nitrocellulose membrane for antibody probing. The membranes were blocked in 5\% milk in tris-buffered saline (TBS) and cut to appropriate size strips for antibody incubation. All antibody incubations occurred at $4{ }^{\circ} \mathrm{C}$ overnight and dilutions occurred in LI-COR blocking buffer (Lincoln, NE) diluted 1:1 with TBS. 
a

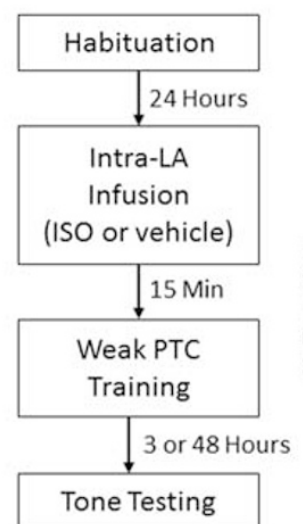

Short-term memory

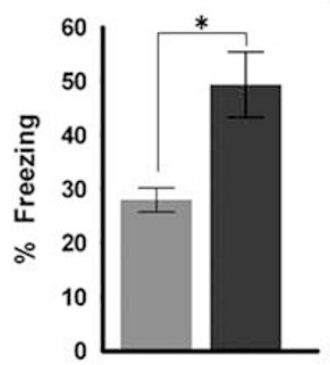

b
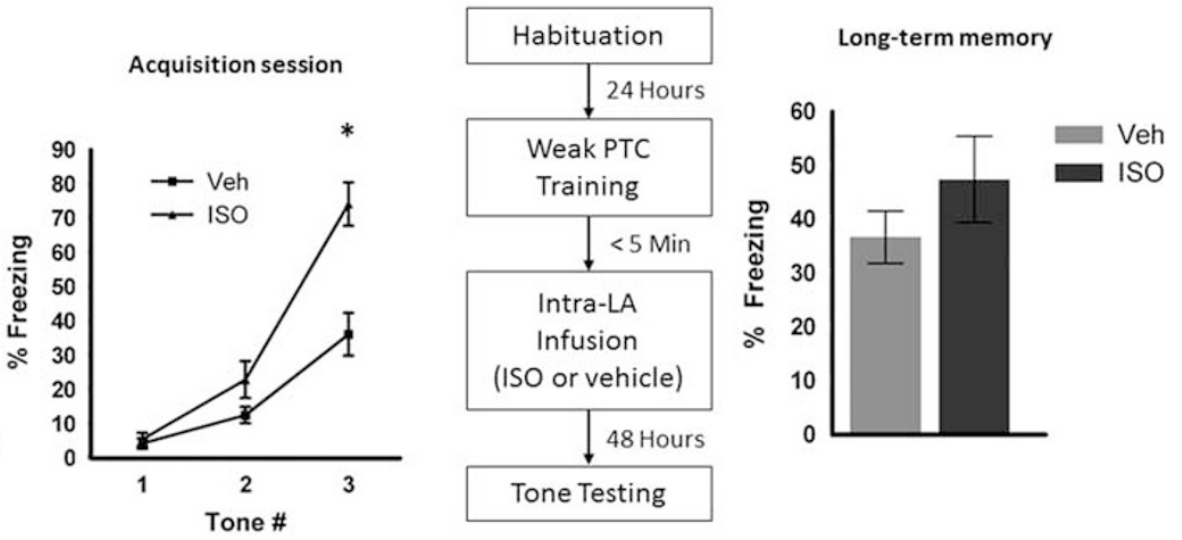

Long-term memory

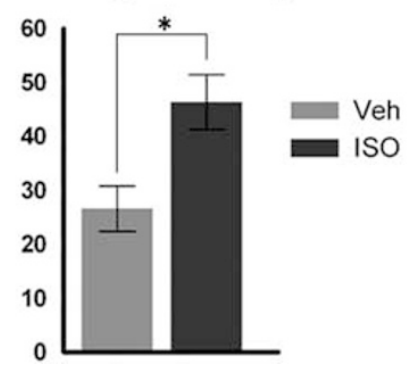

Figure I Pretraining, but not post-training activation of $\beta$ AR with ISO enhances memory formation for PTC. (a) Intra-LA ISO administration I5 min before training enhances freezing responses to the CS at short-term memory measured $3 \mathrm{~h}$ after training and at long-term memory measured $48 \mathrm{~h}$ after training. Enhanced freezing to the CS is also evident during the third CS of the training session. STM ISO $(n=8)$ and vehicle $(n=9)$; LTM ISO $(n=9)$ and vehicle $(n=12)$. (b) Post-training ISO administration does not affect freezing behavior. ISO $(n=8)$ and vehicle $(n=8)$. Error bars indicate \pm SEM. * $p<0.05$.

\section{Data Analysis}

All data are presented as mean \pm SEM. Detailed description of data analyses can be found in the Supplementary Methods.

\section{RESULTS}

\section{$\beta$ AR Activation Facilitates the Acquisition of Aversive Pavlovian Memory Formation}

We tested the hypothesis that pharmacological activation of $\beta$ ARs in LA enhances aversive memory formation. We used a weak PTC training protocol (Rogan et al, 1997) in which animals were exposed to three pairings of an auditory CS coterminating with a weak US ( $0.4 \mathrm{~mA}$ footshock), resulting in $30-40 \%$ CS-evoked freezing (Figure 1). This protocol was chosen to avoid ceiling levels of freezing to allow for the investigation of memory enhancement, measured as increased freezing. Animals receiving the $\beta \mathrm{AR}$ agonist ISO infused into the LA before PTC training displayed higher levels of CS-evoked freezing during the training session compared to vehicle infusion, indicating that $\beta \mathrm{AR}$ activation strengthened learning (Figure 1a). A two-way repeated measures ANOVA revealed a significant interaction between time (CS number) and drug treatment (ISO vs vehicle; $\mathrm{F}$ $(2,38)=8.059 ; p=0.0012)$, and post hoc $t$-tests revealed that CS-evoked freezing was higher in animals treated with ISO during the third CS $(\mathrm{df}=19 ; t=4.20 ; p=0.0005)$.

This enhanced freezing persisted into a STM test conducted $3 \mathrm{~h}$ after training or a LTM test $48 \mathrm{~h}$ after training. In separate groups of animals, we found that ISO infusion prior to training led to an increase in CS-evoked freezing at both STM (two-tailed unpaired $t$-test $\mathrm{df}=15$, $t=3.458, \quad p=0.0035$ ) and LTM (Figure 1a; two-tailed unpaired $t$-test $\mathrm{df}=19, t=3.021, p=0.007)$. A subset of animals tested for STM was re-tested $48 \mathrm{~h}$ later for LTM. The subgroup of animals treated with ISO also displayed enhanced CS-evoked freezing in the LTM test $(\mathrm{df}=10$, $t=2.304, p=0.044$; not shown), indicating that the enhanced memory observed for STM persisted to LTM. This finding was not attributable to an effect of ISO on memory retrieval or expression because freezing was unaffected by pre-testing ISO infusion (Supplementary Results; Supplementary Figure S2A), and because enhanced freezing in ISO-treated animals was observed later under drug-free conditions at LTM. Further, intra-LA ISO administration did not affect the animals' sensitivity to the electrical footshock US (Supplementary Results; Supplementary Figure S2B). Thus, we conclude that the enhanced memory observed during testing was attributable to an effect on memory formation.

We tested whether the effects of ISO on LTM might reflect consolidation processes occurring in the minutes to hours following learning by infusing ISO into the LA immediately after PTC training. Such post-training manipulation procedures have been the gold standard for testing consolidation mechanisms (McGaugh, 1966; McGaugh and Roozendaal, 2009). We found that post-training ISO did not affect freezing during the LTM test (Figure 1b; two-tailed unpaired $t$-test, $\mathrm{df}=14 ; t=1.144 ; p=0.27)$. Because both STM and 
LTM are enhanced by pretraining ISO administration, and post-training ISO administration does not affect memory formation, we conclude that ISO's effects on memory formation are specific to acquisition processes.

\section{Rapid CP-AMPAR Activity, Recruited by $\beta$ ARs, is Required for Memory Acquisition}

Previous work has shown that AMPAR phosphorylation and CP-AMPAR trafficking occur during memory formation for PTC, and these mechanisms are required for learning (Esteban et al, 2003; Rumpel et al, 2005; Humeau et al, 2007; $\mathrm{Hu}$ et al, 2007). $\beta$ ARs may modulate AMPARs through $\mathrm{G}_{\mathrm{s}}$-dependent activation of PKA, which phosphorylates the GluA1 subunit of AMPARs at a serine residue (S845; Winder and Conn, 1993; Esteban et al, 2003), leading to rapid insertion of GluA1-containing CP-AMPARs into the synaptic membrane (Ehlers, 2000; Esteban et al, 2003; Plant et al, 2006; Hu et al, 2007; Tenorio et al, 2010). Indeed, phosphorylation at S845 is a necessary step for plasticity (Goel et al, 2011). Therefore, $\beta$ AR-mediated phosphorylation of S845 GluA1 within the LA may be required for early phases of memory formation.

We first tested the effect of $\beta$ AR activation on S845 GluA1 phosphorylation. Animals received intra-LA infusions of ISO or vehicle, and LA tissue was microdissected and processed for western blot analysis. Phosphorylated S845 GluA1 protein was significantly elevated in animals treated with ISO 15 min after the infusion (Figure 2a) with no change in total levels of GluA1 (two-tailed unpaired $t$-test for ISO $v s$ vehicle; $\mathrm{df}=5 ; t=2.776 ; p=0.0113$ ), confirming that $\beta \mathrm{AR}$ activity is sufficient to phosphorylate S845 GluAl in the LA.

Because ISO infusions in LA enhanced acquisition (Figure 1), we tested whether $\beta \mathrm{AR}$ activity and weak PTC synergistically increase S845 GluA1 phosphorylation. Animals received intra-LA infusions of ISO or vehicle followed by either weak PTC training or a behavioral control condition in which animals were exposed to the training chamber but received no stimuli (box control). LA tissue was microdissected and processed for western blot analysis 5 or $20 \mathrm{~min}$ after the end of the training session. At 5 min posttraining, a two-way ANOVA revealed a main effect of drug such that S845 phosphorylation was increased in animals treated with ISO $(\mathrm{F}(1,39)=5.107 ; p=0.0295)$. There was no effect of behavioral protocol (weak PTC vs box; $\mathrm{F}(1,39)=0.7149 ; \quad p=0.4030)$, nor an interaction $(\mathrm{F}(1,39)=0.01351 ; p=0.9080$; Figure $2 \mathrm{~b})$. This effect was eliminated $20 \mathrm{~min}$ after PTC training (main effect of drug: F $(1,22)=0.1522 ; \quad p=0.7001 ;$ main effect of behavior: $F$ $(1,22)=0.1009 ; \quad p=0.7538 ;$ interaction: $\mathrm{F}(1,22)=0.02732$; $p=0.8702$ ). Thus, at early time points, $\beta$ AR stimulation led to S845 GluA1 phosphorylation in the LA, with no additional increases in phosphorylation following weak PTC. This phosphorylation is a necessary step in CP-AMPAR insertion, which occurs within $5 \mathrm{~min}$ of training on a strong PTC protocol (Hong et al, 2013), indicating that CP-AMPAR function may contribute to the increase in freezing seen with ISO infusion.

We thus tested the hypothesis that $\beta$ AR activation in LA promotes acquisition through increases in CP-AMPAR activity by infusing NASPM (an inhibitor of CP-AMPARs) or vehicle followed by an additional infusion of ISO or vehicle before PTC training (Figure 3a). At STM, ISOenhanced freezing (two-way ANOVA: main effect of ISO $\mathrm{F}(1,41)=5.2 ; p=0.0279)$, while NASPM had no effect on freezing (two-way ANOVA: main effect of NASPM $(\mathrm{F}(1,41)=0.678 ; p=0.415)$, and there was a significant interaction between treatment groups $(\mathrm{F}(1,41)=19.71$; $p=0.0001)$. Post hoc Tukey's multiple comparisons tests confirmed that animals treated with ISO alone froze significantly more compared with vehicle alone $(p=0.0002)$ and ISO+NASPM $(p=0.0016)$. The comparison between the ISO alone group and the NASPM alone group approached significance $(p=0.119)$. As previously seen, animals treated with ISO froze significantly more than those in other treatment groups and NASPM prevented this enhancement (Figure $3 \mathrm{~b}$ ), indicating that CP-AMPARs are necessary for the increase in STM freezing caused by pretraining $\beta \mathrm{AR}$ stimulation.

We tested the same animals $48 \mathrm{~h}$ after training for CS-evoked freezing during LTM. Because of the weak nature of the PTC protocol, we observed a reduction in freezing from the STM session. Despite this reduction, animals given ISO alone maintained elevated freezing compared with animals given vehicle alone (Figure 3c). A two-way ANOVA revealed a significant interaction between animals treated with or without ISO compared with or without NASPM $(\mathrm{F}(1,36)=4.525 ; p=0.040)$. A main effect of ISO or NASPM was not observed (for ISO $\mathrm{F}(1,36) ; p=0.061$; for NASPM F $(1,36)=2.454 ; p=0.126)$. Post hoc Tukey's multiple comparisons confirmed that animals treated with ISO alone froze significantly more compared to vehicle alone $(p=0.0327)$ and ISO+NASPM $(p=0.0293)$. The comparison between the ISO alone group and the NASPM alone group approached significance $(p=0.0808)$. Therefore, $\beta$ ARmediated enhancement in freezing at LTM time points depends on the availability of CP-AMPARs. Altogether, our results show that during acquisition, $\beta \mathrm{AR}$ activity phosphorylates S845 GluA1, likely leading to CP-AMPAR insertion during PTC training, and these CP-AMPARs are required for $\beta$ AR-mediated enhanced freezing at both STM and LTM. We suggest that $\beta$ AR-mediated $\mathrm{S} 845$ phosphorylation primes GluA1-containing CP-AMPARs for synaptic insertion during PTC training.

We noted that animals treated with NASPM alone or NASPM+ISO froze at levels equivalent to the vehicle group at both STM and LTM tests (Figure 3). This finding indicates that memory formation following weak PTC training occurs normally in the absence of CP-AMPARs, whereas $\beta$ AR-mediated enhanced memory requires CP-AMPARs. Together with previous findings (Rumpel et al, 2005; Ganea et al, 2015), this result indicates that strong, but not weak memory formation requires CP-AMPARs.

\section{Delayed ERK Activity, Initiated by $\beta$ AR Activity During Training, is Required for Memory Consolidation}

In addition to signaling to CP-AMPARs, $\beta$ AR signaling is known to activate molecular targets involved in consolidation processes such as ERK, which is required for memory consolidation as evidenced by impairments in LTM, but not STM when antagonists are infused into the LA either before or immediately after training (Meitzen et al, 2011; Schafe et al, 2000). We previously showed that post-training 
a

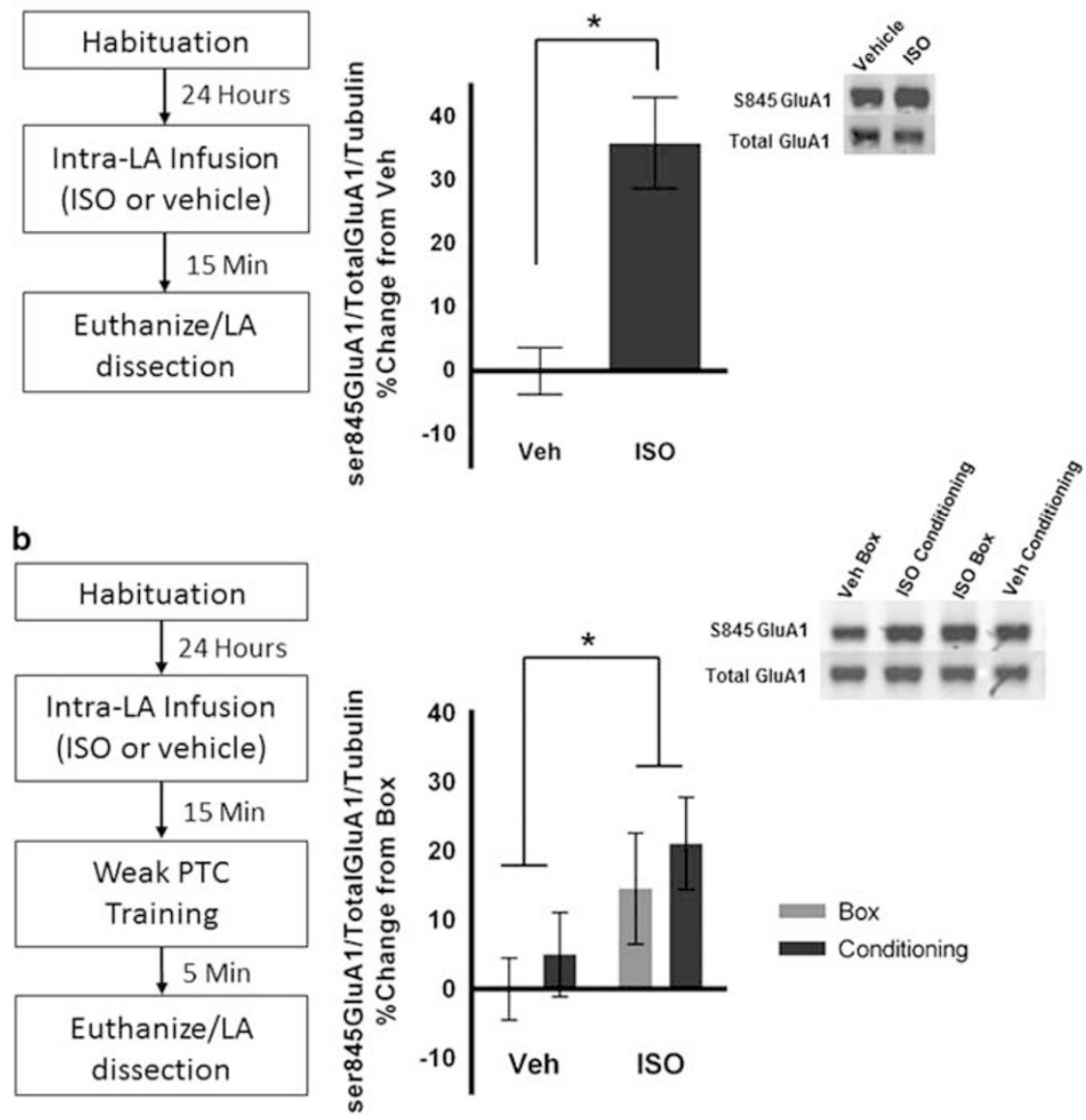

Figure $2 \beta A R$ activation leads to GluAI phosphorylation at S845. (a) Bilateral intra-LA infusion of ISO $(n=4)$ increases GluAI phosphorylation at S845 compared with vehicle $(n=3)$. All values are normalized to the loading control $\alpha$-tubulin. ISO values are presented as a percent increase from the control condition. (b) Bilateral intra-LA ISO administration enhances GluAI S845 phosphorylation in all animals independent of learning 5 min after weak PTC training. Training for weak PTC does not induce GluAI S845 phosphorylation. For 5 min, vehicle box $(n=9)$, vehicle conditioning $(n=12)$, ISO box $(n=12)$, ISO conditioning $(n=10)$. Insets show sample bands from each condition. Error bars represent \pm SEM. $* p<0.05$.

a

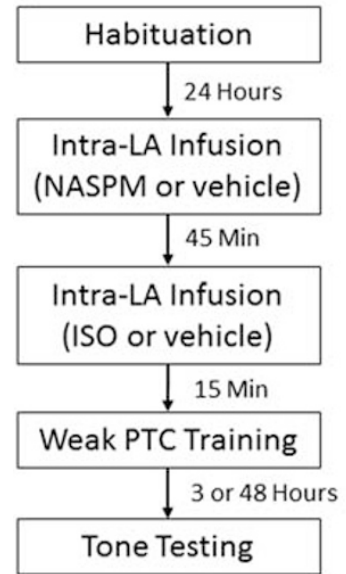

b

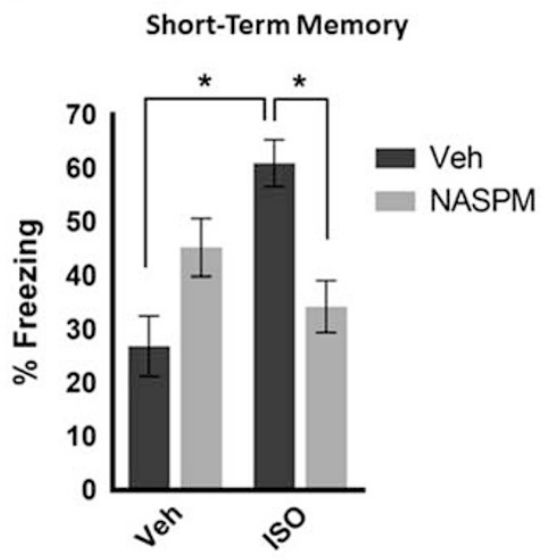

C

\section{Long-Term Memory}

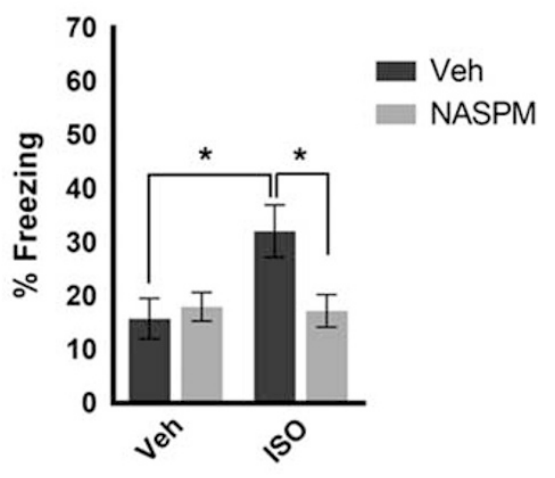

Figure 3 Enhanced short-term and long-term memory by ISO administration depends on CP-AMPARs. (a) Timeline of experimental procedures. (b) Animals treated with ISO alone $(n=13)$ show significantly enhanced freezing at STM measured $3 \mathrm{~h}$ after training compared with vehicle or ISO+NASPM: vehicle $(n=9)$, ISO+NASPM $(n=12)$, NASPM alone $(n=11)$. (c) Animals treated with ISO alone $(n=12)$ show significantly enhanced freezing at LTM measured $48 \mathrm{~h}$ after training compared with vehicle or ISO+NASPM: vehicle $(n=8)$, ISO+NASPM $(n=12)$, NASPM alone $(n=8)$. Error bars represent \pm SEM. ${ }^{*} p<0.05$. 
a

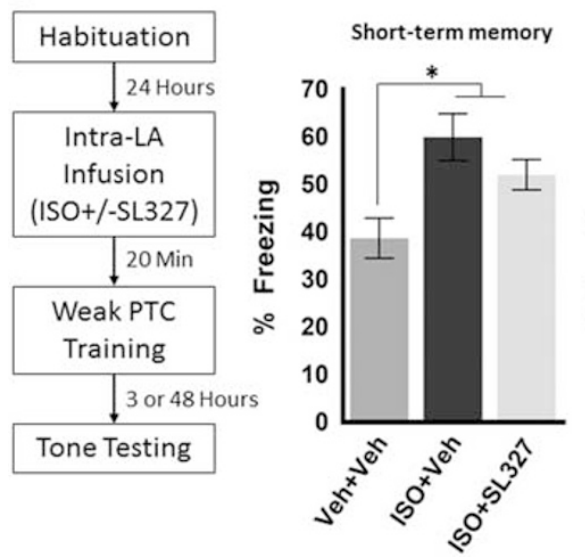

b

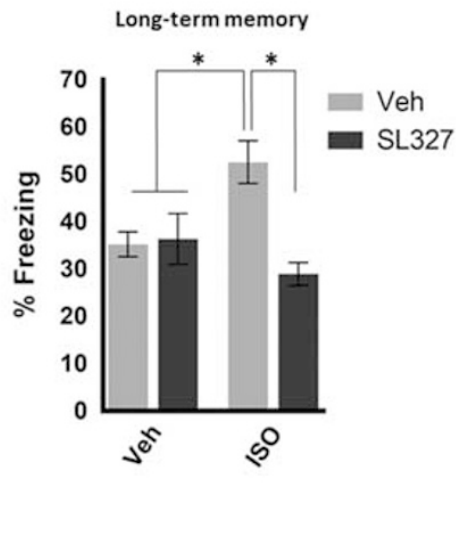

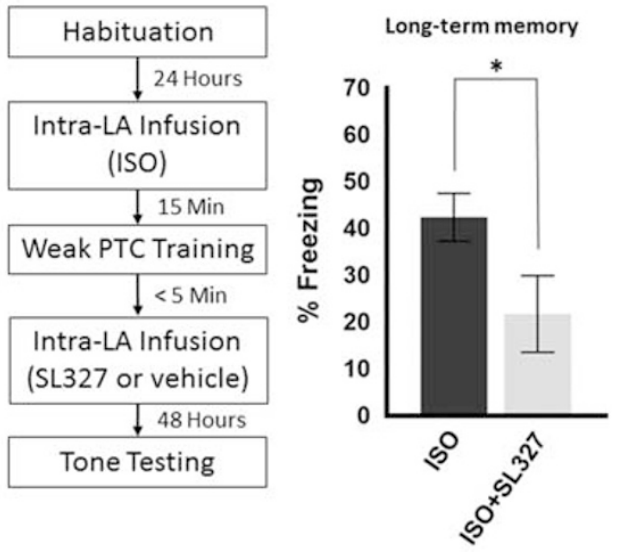

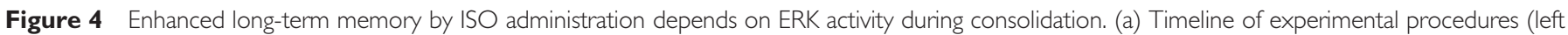

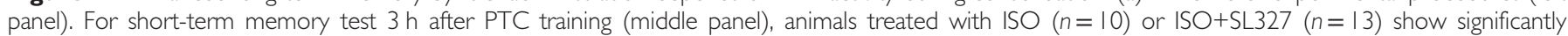

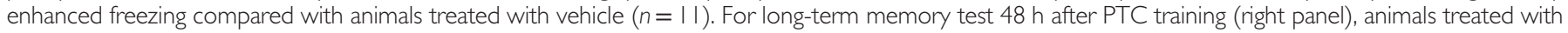

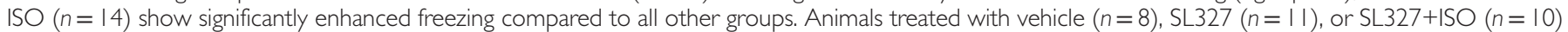

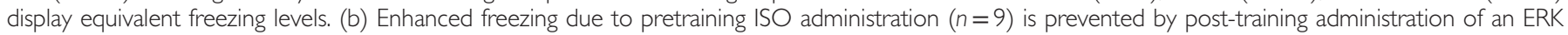
inhibitor $(n=6)$. Error bars represent \pm SEM. $* p<0.05$.

infusion of the $\beta \mathrm{AR}$ antagonist, propranolol, did not impair memory consolidation (Bush et al, 2010), suggesting that $\beta \mathrm{ARs}$ modulate learning only during acquisition. If $\beta \mathrm{AR}-$ mediated activation of ERK is required for consolidation processes, then this process must be initiated during acquisition. We therefore tested the hypothesis that although ERK is recruited by $\beta \mathrm{AR}$ activity during training, its activity is required later during the consolidation phase for enhanced LTM. We first confirmed previous findings that $\beta \mathrm{AR}$ activation by ISO leads to ERK activity by examining neurons positive for phosphorylated ERK (pERK) following an infusion of ISO or vehicle into the LA at early and late time points (Supplementary Figure S3A). Fifteen minutes after infusion, we observed no change in pERK levels (twotailed paired $t$-test $\mathrm{df}=6 ; t=0.9012 ; p=0.402)$. Forty-five minutes after infusion, we observed a significant increase in pERK levels (two-tailed paired $t$-test $\mathrm{df}=5 ; t=2.845$; $p=0.0360)$.

For behavioral experiments, we first used a strong training protocol to confirm the effectiveness of SL327, a drug that prevents ERK activation. Indeed, SL327 effectively reduced CS-evoked freezing during LTM test (Supplementary Figure S3B; two-tailed unpaired $t$-test $\mathrm{df}=13 ; t=2.842$; $p=0.014)$. We then tested whether ERK is involved in $\beta$ ARmediated memory enhancement of STM by infusing ISO alone or in combination with SL327 before training. A oneway ANOVA revealed a significant difference in CS-evoked freezing between treatment groups for the STM test (Figure 4a; $\mathrm{F}=6.613 ; p=0.0041$ ), and post hoc Dunnett's multiple comparison tests revealed that animals treated with ISO or ISO+SL327 froze significantly more than animals treated with vehicle (ISO $v s$ vehicle $p=0.002$; ISO+SL327 vs vehicle $p=0.042$ ).

Although not required for STM, we found that ERK activity is required for $\beta \mathrm{AR}$-mediated enhanced freezing at LTM. For examining LTM, we included an additional control group in which SL327 was administered alone, for a total of four drug groups. We included this group because
ERK inhibition has been shown to interfere with formation of LTM but not STM (Schafe et al, 2000). A two-way ANOVA revealed a significant interaction between treatment groups: animals treated with or without ISO compared with animals treated with or without $\operatorname{SL327}(\mathrm{F}(1,39)=7.907$; $p=0.0077)$. Post hoc Tukey's multiple comparisons tests revealed that animals in the ISO alone group froze significantly more than animals in every other group (Figure 4a; ISO vs vehicle: $p=0.0407$; ISO vs SL327: $p=0.0333$; ISO $v s$ ISO+SL327: $p=0.0013$ ), whereas all other groups exhibited equivalent levels of freezing (vehicle $v s$ SL327: $p=0.8724$; vehicle vs ISO+SL327: $p=0.1011$; SL327 vs ISO+SL327: $p=0.2421)$. Altogether, these results suggest that ERK activity is necessary for ISO-enhanced freezing at LTM but not STM.

$\beta \mathrm{AR}$ activity occurs during acquisition, but our results suggest that ERK activity is not required during acquisition or STM. To explicitly test the notion that ERK activity occurs during the consolidation period despite initial activation during training, we conducted an experiment in which animals received an intra-LA infusion of ISO before training and an intra-LA infusion of SL327 or vehicle immediately after training. Because administration of SL327 had no effect on STM, freezing levels were only evaluated at the LTM time point. Consistent with Figure $4 \mathrm{a}$, animals treated with ISO pretraining and SL327 post-training showed lower freezing levels than animals treated with pretraining ISO and posttraining vehicle (Figure $4 \mathrm{~b}$; one-tailed unpaired $t$-test $\mathrm{df}=13$, $t=2.26, p=0.021)$. Importantly, the acquisition curve for these groups did not differ (Supplementary Figure S3C). Together with the null effect of post-training ISO infusion (Figure 1), these results suggest that $\beta \mathrm{AR}$ activity during learning initiates a signaling cascade leading to later ERK activity during consolidation.

Again, we noted that animals treated with SL327 showed equivalent CS-evoked freezing compared with those treated with vehicle at LTM test (Figure 4a), indicating that LTM formation following weak training does not require ERK 
activity. This suggests that alternative signaling pathways may compensate for ERK blockade when memory strength is weak. Strong memory formation, whether produced from training with a strong shock or pharmacologically with ISO, requires ERK activity (Figure 4; Schafe et al, 2000).

\section{DISCUSSION}

Previous work has shown that $\beta \mathrm{AR}$ activity within the LA is required for memory formation when training with a strong US (Bush et al, 2010). Here we show that $\beta$ AR stimulation in the LA enhances memory formation through temporal regulation of specific intracellular mechanisms. Using a weak PTC protocol, we found that pharmacological activation of $\beta$ ARs in the LA before training enhances freezing during the training session, STM, and LTM tests, whereas infusions immediately after training had no effect. These results indicate that engagement of $\beta$ ARs specifically during the acquisition phase enhances memory formation. The weak shock used in the current studies causes sub-maximal NE release relative to stronger shocks typically used in PTC studies (Quirarte et al, 1998). Thus, ISO administration may enhance aversive associative learning by engaging additional $\beta$ ARs and priming plasticity-related proteins such as CP-AMPARs and ERK.

We uncover a functional link between surface-expressed $\beta \mathrm{ARs}$ and their intracellular signaling targets in vivo, during a learning event, along with the temporal dynamics of these molecules following the learned event. Specifically, $\beta \mathrm{AR}$ activity in the LA during acquisition modulates memory formation by initiating an intracellular signaling cascade that recruits at least two distinct targets associated with discrete phases of Pavlovian learning. These mechanisms may contribute to strengthening of CS input synapses to neurons in the LA (Bailey et al, 2000; Johansen et al, 2014; Sears et al, 2014).

In the initial moments after $\beta \mathrm{AR}$ stimulation, GluA1 subunits are rapidly phosphorylated at the S845 site (Figure 2a). This step may permit synaptic incorporation of CP-AMPARs and enhance their functionality (Malinow and Malenka, 2002). Engaging these AMPARs is a critical process in $\beta$ AR-mediated acquisition indicated by reduced freezing during STM (to control levels) when the CP-AMPAR blocker NASPM was infused along with ISO. These results are consistent with previous findings that $\beta$ ARs, via PKA, phosphorylate S845 GluA1 (Banke et al, 2000; Esteban et al, 2003), that NE regulates CP-AMPAR activity and trafficking ( $\mathrm{Hu}$ et al, 2007), and that CP-AMPAR synaptic incorporation is required for plasticity and memory formation in the amygdala following PTC (Rumpel et al, 2005; Humeau et al, 2007). Thus, we propose that ISO-induced phosphorylation of GluA1 at S845 primes CP-AMPARs for synaptic insertion during CS-US pairings and permits robust learning in response to a weak US.

Clem and Huganir (2010) found normal PTC memory formation in a mutant mouse in which the S845 residue of GluA1 is mutated to an alanine, prohibiting PKA-mediated phosphorylation. In the absence of the S845 site, CP-AMPARs were inserted normally into the synaptic membrane following PTC, and learning was intact. Our studies using rats reveal that ISO infusion rapidly phosphorylated S845 GluA1 and that CP-AMPAR activity is required for enhanced memory formation following weak PTC training combined with ISO infusion. This discrepancy may suggest the engagement of redundant mechanisms underlying learning in the mutant mouse model. Alternatively, the pharmacological stimulation of $\beta$ ARs may cause more robust S845 phosphorylation than PTC training alone.

We further found that $\beta \mathrm{AR}$-mediated enhancement of LTM was dependent on ERK, consistent with ERK's established role in memory consolidation (Schafe et al, 2000). Specifically, $\beta$ AR activity during acquisition, through a sustained signaling pathway, leads to the later recruitment of ERK during consolidation. Once recruited downstream of $\beta$ ARs, ERK may stabilize plasticity by inducing gene transcription and protein synthesis (Bourtchuladze et al, 1994; Bailey et al, 1999; Schafe and LeDoux, 2000). Consistent with previous findings in vitro (Huang and Kandel, 1996; Gelinas et al, 2007), our results show that $\beta$ ARs recruit ERK activity during aversive learning and that this process is required for robust LTM. Intriguingly, a similar finding has been reported for PTC in the serotonergic system, in which serotonin released during acquisition in previously stressed animals is required for later memory consolidation (Baratta et al, 2016).

Altogether, our results show that $\beta \mathrm{AR}$-mediated enhanced memory formation requires $\mathrm{CP}$-AMPAR recruitment for acquisition and ERK recruitment for consolidation processes, indicating that $\mathrm{NE}$ engagement recruits these processes during learning (Supplementary Figure S4). We further found that weak memory formation does not require CP-AMPARs or ERK, unlike formation of salient memories involving a strong shock US (Schafe et al, 2000; Rumpel et al, 2005), indicating that weak memory formation may rely on alternative molecular signaling pathways. Which molecules participate in memory formation may be determined by the degree of NE released into the LA, which varies as a function of shock intensity (Quirarte et al, 1998). The shock intensity, in turn, determines the magnitude of freezing, particularly when the CS is always paired with the US, a measurement that indicates memory strength (Rescorla and Wagner, 1972). This behavioral phenomenon may be explained by increasing shock intensities promoting stronger $\beta \mathrm{AR}$ engagement together with $\beta \mathrm{AR}$-dependent recruitment of plasticity-related proteins such as CP-AMPARs and ERK.

Although we found that $\beta \mathrm{AR}$ activation with ISO only enhances memory when administered before training, other aversive learning tasks such as inhibitory avoidance, which has instrumental and contextual components, require $\beta \mathrm{AR}$ activation in the consolidation phase, indicated by effective post-training manipulations (Haycock et al, 1976; McGaugh, 2002). This discrepancy may occur because distinct neural circuitry supports learning in each task. In cued PTC, both acquisition and consolidation depend on the amygdala (Rogan et al, 1997; Blair et al, 2001). In contrast, memory consolidation for inhibitory avoidance depends on the hippocampus (Da Cunha et al, 1991), and the amygdala modulates these storage processes (McGaugh, 2002). Direct comparison of amygdala manipulations on these tasks reveals such key differences (Wilensky et al, 1999, 2000). For cued PTC, we conclude that $\beta \mathrm{AR}$ activity occurs specifically during learning, contributing directly to acquisition processes, and indirectly to consolidation processes by 
signaling to persistently active molecules. This finding indicates that $\beta \mathrm{ARs}$ contribute to the consolidation phase of cued PTC in a manner that is not captured by studies only using post-training manipulations.

In summary, we have linked two distinct molecular targets to their surface-expressed noradrenergic receptor, which participate in modulation of aversive memory formation in the amygdala. Our data suggest that these targets are dissociable by their timing properties: CP-AMPARs are recruited for CS-US associations during learning while ERK stabilizes these associations into a long-term memory. Thus, NE modulates both acquisition and consolidation through distinct signaling cascades. These results may explain the limited efficacy of pharmacological treatments targeting the NE system when administered following a traumatic event (eg, Albucher and Liberzon, 2002; Pitman et al, 2002) because signaling elements involved in memory consolidation may have already been initiated. Our results support the idea that elevated $\mathrm{NE}$ and $\beta \mathrm{AR}$ activity within the amygdala may enhance acquisition and consolidation for events that closely follow a salient or traumatic experience.

\section{FUNDING AND DISCLOSURE}

Research supported by an NIMH grant to JEL. (R01 MH046516). The authors declare no conflict of interest.

\section{ACKNOWLEDGMENTS}

We thank C.R. Farb, J.M. Moscarello, and E.C. Andrade for technical assistance. We also thank T.J. Carew and N. Heintz for sharing equipment.

\section{REFERENCES}

Albucher RC, Liberzon I (2002). Psychopharmacological treatment in PTSD: a critical review. J Psychiatr Res 36: 355-367.

Bailey DJ, Kim JJ, Sun W, Thompson RF, Helmstetter FJ (1999). Acquisition of fear conditioning in rats requires the synthesis of mRNA in the amygdala. Behav Neurosci 113: 276-282.

Bailey CH, Giustetto M, Huang YY, Hawkins RD, Kandel ER (2000). Is heterosynaptic modulation essential for stabilizing Hebbian plasticity and memory? Nat Rev Neurosci 1: 11-20.

Banke TG, Bowie D, Lee H, Huganir RL, Schousboe A, Traynelis SF (2000). Control of GluR1 AMPA receptor function by cAMPdependent protein kinase. J Neurosci 20: 89-102.

Baratta MV, Kodandaramaiah SB, Monahan PE, Yao J, Weber MD, Lin A et al (2016). Stress enables reinforcement-elicited serotonergic consolidation of fear memory. Biol Psychiatry 79: 814-822.

Berridge CW, Waterhouse BD (2003). The locus coeruleusnoradrenergic system: modulation of behavioral state and statedependent cognitive processes. Brain Res Brain Res Rev 42: 33-84.

Blair HT, Schafe GE, Bauer EP, Rodrigues SM, LeDoux JE (2001). Synaptic plasticity in the lateral amygdala: a cellular hypothesis of fear conditioning. Learn Mem 8: 229-242.

Bourtchuladze R, Frenguelli B, Blendy J, Cioffi D, Schutz G, Silva AJ (1994). Deficient long-term memory in mice with a targeted mutation of the cAMP-responsive element-binding protein. Cell 79: 59-68.

Bush DE, Caparosa EM, Gekker A, Ledoux J (2010). Betaadrenergic receptors in the lateral nucleus of the amygdala contribute to the acquisition but not the consolidation of auditory fear conditioning. Front Behav Neurosci 4: 154.
Chen FJ, Sara SJ (2007). Locus coeruleus activation by foot shock or electrical stimulation inhibits amygdala neurons. Neuroscience 144: 472-481.

Clem RL, Huganir RL (2010). Calcium-permeable AMPA receptor dynamics mediate fear memory erasure. Science 330: 1108-1112.

Da Cunha C, Wolfman C, Huang CH, Walz R, Koya R, Bianchin M et al (1991). Effect of post-training injections of flumazenil into the amygdala, hippocampus and septum on retention of habituation and of inhibitory avoidance in rats. Braz J Med Biol Res 24: 301-306.

Ding X, Qiao Y, Piao C, Zheng X, Liu Z, Liang J (2014). N-methyl$\mathrm{D}$-aspartate receptor-mediated glutamate transmission in nucleus accumbens plays a more important role than that in dorsal striatum in cognitive flexibility. Front Behav Neurosci 8: 304.

Ehlers MD (2000). Reinsertion or degradation of AMPA receptors determined by activity-dependent endocytic sorting. Neuron 28: 511-525.

Ennis M, Aston-Jones G, Shiekhattar R (1992). Activation of locus coeruleus neurons by nucleus paragigantocellularis or noxious sensory stimulation is mediated by intracoerulear excitatory amino acid neurotransmission. Brain Res 598: 185-195.

Esteban JA, Shi SH, Wilson C, Nuriya M, Huganir RL, Malinow R (2003). PKA phosphorylation of AMPA receptor subunits controls synaptic trafficking underlying plasticity. Nat Neurosci 6: 136-143.

Faber ES, Delaney AJ, Power JM, Sedlak PL, Crane JW, Sah P (2008). Modulation of SK channel trafficking by beta adrenoceptors enhances excitatory synaptic transmission and plasticity in the amygdala. J Neurosci 28: 10803-10813.

Fanselow MS, Poulos AM (2005). The neuroscience of mammalian associative learning. Annu Rev Psychol 56: 207-234.

Fendt M, Koch M, Schnitzler HU (1994). Amygdaloid noradrenaline is involved in the sensitization of the acoustic startle response in rats. Pharmacol Biochem Behav 48: 307-314.

Ganea DA, Dines M, Basu S, Lamprecht R (2015). The membrane proximal region of AMPA receptors in lateral amygdala is essential for fear memory formation. Neuropsychopharmacology 40: 2727-2735.

Gelinas JN, Banko JL, Hou L, Sonenberg N, Weeber EJ, Klann E et al (2007). ERK and mTOR signaling couple beta-adrenergic receptors to translation initiation machinery to gate induction of protein synthesis-dependent long-term potentiation. J Biol Chem 282: 27527-27535.

Goel A, Xu LW, Snyder KP, Song L, Goenaga-Vazquez Y, Megill A et al (2011). Phosphorylation of AMPA receptors is required for sensory deprivation-induced homeostatic synaptic plasticity. PLOS ONE 6: e18264.

Hatfield T, McGaugh JL (1999). Norepinephrine infused into the basolateral amygdala posttraining enhances retention in a spatial water maze task. Neurobiol Learn Mem 71: 232-239.

Haycock JW, van Buskirk R, McGaugh JL (1976). Facilitation of retention performance in mice by posttraining diethyldithiocarbamate. Pharmacol Biochem Behav 5: 525-528.

Hong I, Kim J, Kim J, Lee S, Ko HG, Nader K et al (2013). AMPA receptor exchange underlies transient memory destabilization on retrieval. Proc Natl Acad Sci USA 110: 8218-8223.

$\mathrm{Hu} \mathrm{H}$, Real E, Takamiya K, Kang MG, LeDoux J, Huganir RL et al (2007). Emotion enhances learning via norepinephrine regulation of AMPA-receptor trafficking. Cell 131: 160-173.

Huang YY, Kandel ER (1996). Modulation of both the early and the late phase of mossy fiber LTP by the activation of beta-adrenergic receptors. Neuron 16: 611-617.

Humeau Y, Reisel D, Johnson AW, Borchardt T, Jensen V, Gebhardt C et al (2007). A pathway-specific function for different AMPA receptor subunits in amygdala long-term potentiation and fear conditioning. J Neurosci 27: 10947-10956.

Johansen JP, Diaz-Mataix L, Hamanaka H, Ozawa T, Ycu E, Koivumaa $\mathrm{J}$ et al (2014). Hebbian and neuromodulatory 
mechanisms interact to trigger associative memory formation. Proc Natl Acad Sci USA 111: E5584-E5592.

LeDoux JE (2014). Coming to terms with fear. Proc Natl Acad Sci USA 111: 2871-2878.

Malinow R, Malenka RC (2002). AMPA receptor trafficking and synaptic plasticity. Annu Rev Neurosci 25: 103-126.

McGaugh JL (1966). Time-dependent processes in memory storage. Science 153: 1351-1358.

McGaugh JL (2002). Memory consolidation and the amygdala: a systems perspective. Trends Neurosci 25: 456.

McGaugh JL, Roozendaal B (2009). Drug enhancement of memory consolidation: historical perspective and neurobiological implications. Psychopharmacology (Berl) 202: 3-14.

McKernan MG, Shinnick-Gallagher P (1997). Fear conditioning induces a lasting potentiation of synaptic currents in vitro. Nature 390: 607-611.

Meitzen J, Luoma JI, Stern CM, Mermelstein PG (2011). beta1Adrenergic receptors activate two distinct signaling pathways in striatal neurons. J Neurochem 116: 984-995.

Murchison CF, Zhang XY, Zhang WP, Ouyang M, Lee A, Thomas SA (2004). A distinct role for norepinephrine in memory retrieval. Cell 117: 131-143.

Pitman RK, Sanders KM, Zusman RM, Healy AR, Cheema F, Lasko NB et al (2002). Pilot study of secondary prevention of posttraumatic stress disorder with propranolol. Biol Psychiatry 51: 189-192.

Plant K, Pelkey KA, Bortolotto ZA, Morita D, Terashima A, McBain CJ et al (2006). Transient incorporation of native GluR2lacking AMPA receptors during hippocampal long-term potentiation. Nat Neurosci 9: 602-604.

Quirarte GL, Galvez R, Roozendaal B, McGaugh JL (1998). Norepinephrine release in the amygdala in response to footshock and opioid peptidergic drugs. Brain Res 808: 134-140.

Rescorla RA, Wagner AR (1972). A theory of Pavlovian conditioning: variations in the effectiveness of reinforcement and nonrecinforcement. In: Black AH, Prokasy WF (eds). Classical Conditioning II: Current Research and Theory. Appleton Century Crofts: New York, pp 64-99.

Rodrigues SM, Schafe GE, LeDoux JE (2004). Molecular mechanisms underlying emotional learning and memory in the lateral amygdala. Neuron 44: 75-91.
Rogan MT, Staubli UV, LeDoux JE (1997). Fear conditioning induces associative long-term potentiation in the amygdala. Nature 390: 604-607.

Rumpel S, LeDoux J, Zador A, Malinow R (2005). Postsynaptic receptor trafficking underlying a form of associative learning. Science 308: 83-88.

Schafe GE, Atkins CM, Swank MW, Bauer EP, Sweatt JD, LeDoux JE (2000). Activation of ERK/MAP kinase in the amygdala is required for memory consolidation of pavlovian fear conditioning. J Neurosci 20: 8177-8187.

Schafe GE, LeDoux JE (2000). Memory consolidation of auditory Pavlovian fear conditioning requires protein synthesis and protein kinase A in the amygdala. J Neurosci 20: RC96.

Schulz B, Fendt M, Schnitzler HU (2002). Clonidine injections into the lateral nucleus of the amygdala block acquisition and expression of fear-potentiated startle. Eur J Neurosci 15: 151-157.

Sears RM, Schiff HC, LeDoux JE (2014). Molecular mechanisms of threat learning in the lateral nucleus of the amygdala. Prog Mol Biol Transl Sci 122: 263-304.

Tenorio G, Connor SA, Guévremont D, Abraham WC, Williams J, O'Dell TJ et al (2010). 'Silent' priming of translation-dependent LTP by $ß$-adrenergic receptors involves phosphorylation and recruitment of AMPA receptors. Learn Mem 17: 627-638.

Tully K, Li Y, Tsvetkov E, Bolshakov VY (2007). Norepinephrine enables the induction of associative long-term potentiation at thalamo-amygdala synapses. Proc Natl Acad Sci USA 104: 14146-14150.

Uematsu A, Tan BZ, Johansen JP (2015). Projection specificity in heterogeneous locus coeruleus cell populations: implications for learning and memory. Learn Mem 22: 444-451.

Wilensky AE, Schafe GE, LeDoux JE (1999). The amygdala modulates memory consolidation of fear-motivated inhibitory avoidance learning but not classical fear conditioning. J Neurosci 19: RC48.

Wilensky AE, Schafe GE, LeDoux JE (2000). The amygdala modulates memory consolidation of fear-motivated inhibitory avoidance learning but not classical fear conditioning. J Neurosci 20: 7059-7066.

Winder DG, Conn PJ (1993). Activation of metabotropic glutamate receptors increases cAMP accumulation in hippocampus by potentiating responses to endogenous adenosine. J Neurosci 13: $38-44$.

Supplementary Information accompanies the paper on the Neuropsychopharmacology website (http://www.nature.com/npp) 